\title{
Gastrointestinal non-Hodgkin lymphomas
}

\author{
Magdalena Olszewska-Szopa ${ }^{A-F}$, Tomasz Wróbe ${ }^{A-F}$ \\ Department of Hematology, Blood Neoplasms and Bone Marrow Transplantation, Wroclaw Medical University, Poland \\ A - research concept and design; $B$ - collection and/or assembly of data; $C$ - data analysis and interpretation; \\ $D$ - writing the article; $E$ - critical revision of the article; $F$ - final approval of the article
}

Address for correspondence

Magdalena Olszewska-Szopa

E-mail:molszopa@gmail.com

\section{Funding sources \\ None declared}

\section{Conflict of interest}

None declared

Received on 0ctober 28, 2015

Reviewed on November 23, 2016

Accepted on August 8, 2018

Published online on August 13, 2019

\begin{abstract}
Although gastrointestinal (GI) tract is the most common extranodal site involved in non-Hodgkin lymphoma (NHL), primary gastrointestinal NHL (gNHL) is a rare problem which concerns about 10-15\% of NHL patients and $30-40 \%$ of extranodal NHL patients. Lymphoid neoplasms may consist of mature B, T and (less commonly) extranodal NK/T cells. The most common diagnoses are diffuse large B-cell lymphoma and marginal zonelymphoma (MALT), but many other lymphomas may be found in the Gl tract. There are a few well-known risk factors of gNHL and some of them affect treatment. The most frequent sites of occurrence are the stomach followed by small intestine and ileocecal region. In the last 2 decades, there has been a rapid development in the diagnosis, staging and management of $G$ l lymphoma, but still some of such lymphomas, especially T-cell ones, are a therapeutic challenge. In this review, we present clinical and pathological features of $\mathrm{Gl}$ lymphomas. We also describe the current status in diagnosis and treatment.
\end{abstract}

Key words: DLBCL, gastrointestinal lymphoma, EATL, MALT

Cite as

Olszewska-Szopa M, Wróbel T. Gastrointestinal non-Hodgkin lymphomas. Adv Clin Exp Med. 2019;28(8):1119-1124. doi:10.17219/acem/94068

DOI

10.17219/acem/94068

\section{Copyright}

Copyright by Author(s)

This is an article distributed under the terms of the

Creative Commons Attribution Non-Commercial License

(http://creativecommons.org/licenses/by-nc-nd/4.0/) 


\section{Introduction}

Gastrointestinal (GI) tract is the most common extranodal site involved in non-Hodgkin lymphoma (NHL). Primary gastrointestinal non-Hodgkin lymphoma (gNHL), however, is a rare problem which concerns about 10-15\% of all NHL patients and $30-40 \%$ of extranodal NHL patients. ${ }^{1}$ At the same time, gNHL cases account for only $1-4 \%$ of GI neoplasms. ${ }^{2}$ The most frequent site for gNHL is the stomach (60-75\% of all cases), followed by the small intestine and the ileocecal region (Fig. 1). ${ }^{3}$

Histopathological findings in GI tract reveal indolent as well as aggressive lymphomas, which may consist of mature B, T or NK cells. Intestinal B-cell lymphomas are more frequent than T-cell lymphomas (ratio 6:1). ${ }^{4}$ Two of the most prevalent diagnoses are diffuse large B-cell lymphoma (DLBCL) and marginal zone lymphoma (MALT). Other histologic subtypes - follicular lymphoma (FL), mantle cell lymphoma (MCL), Burkitt lymphoma (BL), enteropathy-associated lymphoma (EATL), post-transplan lymphoproliferative diseases (PTLD), and others - are less commonly observed (Fig. 2). ${ }^{1,3}$

Clinical picture results mainly from localization of the disease, while histopathologic type of the lymphoma is less relevant. The most common symptoms are abdominal pain, nausea, vomiting, diarrhea, and malabsorption. Violent manifestations of the disease in the form of GI bleeding, perforation or intestinal obstruction are not so frequent. ${ }^{1}$

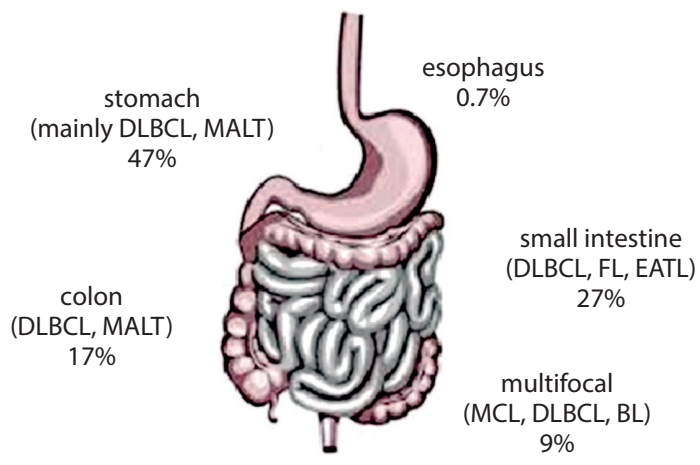

Fig. 1. Gastrointestinal lymphoma topography

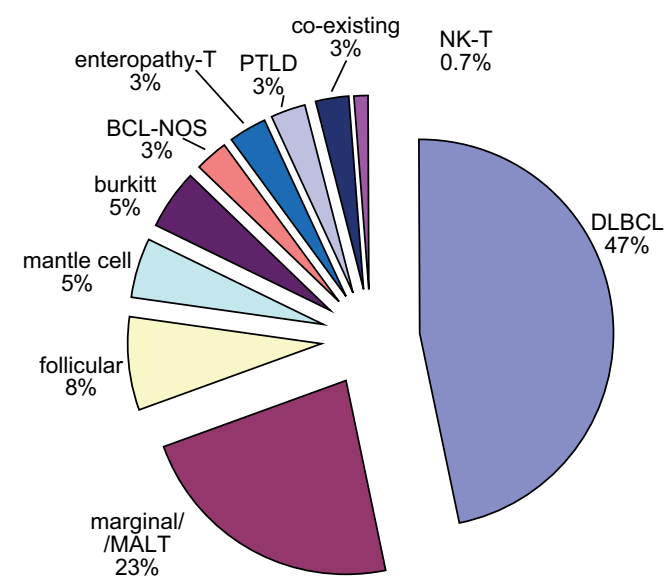

Fig. 2. Gastrointestinal lymphoma distribution

\section{gNHL from top to bottom}

The oropharyngeal region is a location for $2.5 \%$ of NHLs. The most frequently involved area is the Waldeyer's ring. The leading symptoms are dysphagia, hearing loss and pain. The median age at the moment of the diagnosis is above 50 years. The most common histopathological finding is DLBCL. Viral factors are known to increase the risk of nasopharyngeal NHL. Contrary to other neoplasms in this location, chemotherapy and radiotherapy are preferred rather than surgery. The esophagus is an extremely rare location for NHL and primary involvement is casuistic. In the majority of cases, DLBCL is diagnosed. The risk factors are immunodeficiency disorders, particularly HIV. ${ }^{3}$ The stomach is the most commonly involved site in primary gNHL and comprises $60-70 \%$ of gNHL cases. At the same time, it constitutes 3-5\% of all gastric neoplasms. Clinical symptoms are typical for this localization: pain, nausea, emesis, and weight loss. Endoscopic ultrasonography (EUS) is an essential tool in this localization and will be discussed in detail later in the text. Primary small intestine lymphomas account for $20 \%$ of gNHL and $10-20 \%$ of all intestine neoplasms. The most commonly involved region is the ileum. ${ }^{3}$ Histopathological findings reveal the following types of disease: MALT, DLBCL, EATL, MCL, and others. Balloon enteroscopy and capsule endoscopy are among the essential imaging techniques in the diagnosis of all small intestine neoplasms. Some small intestine lymphomas including MCL, FL and MALT, occur in the form of polyps, others appear as firm mass (BL) or nodules, scars and erosions (EATL). ${ }^{3}$ Colorectal lymphoma accounts for approx. 6-12\% of gNHL, but very rarely is the colorectum the primary site for gNHL. In Western countries, lymphomas in this region are of B-cell origin, but in Asia there is an increasing frequency of T-cell-lineage NHL. In some cases of colorectal gNHL, surgery is a treatment as well as a diagnostic tool. ${ }^{3}$

\section{Imaging techniques}

Endoscopy is a fundamental diagnostic technique in gNHLs and it may reveal a wide variety of different forms: from enlarged lymph nodes and lymphoid follicles, which may sometimes appear as reactive, through polyps, to infiltrative and necrotic lesions. ${ }^{1}$ Endoscopic ultrasonography is a very valuable method in locoregional staging; for instance, it allows for the visualization of all layers of gastric walls. Moreover, it shows local lymph nodes. Endoscopic ultrasonography is more valuable in indolent lymphomas, in which locoregional staging is important for proper therapeutic decisions. In aggressive lymphomas, chemo- or immunochemotherapy is usually introduced from the beginning and precise local assessment is not so important. ${ }^{5}$ The impact of EUS in primary gastric 
lymphoma. In the stomach, EUS demonstrates 4 types of patterns: superficially spreading, diffusely infiltrating, mass forming, and mixed. Current stomach MALT classification is based on EUS findings. ${ }^{1}$ Computed tomography (CT) and magnetic resonance imaging (MRI) are valuable in disease assessment outside the GI tube. It is important to remember that a CT scan usually does not enable to visualize lymphoma confined to the mucosa. ${ }^{6} 18 \mathrm{~F}$-fluorodeoxyglucose positron emission tomography (FDG-PET) has proven its usefulness in diagnosis and staging of the disease and in response assessment. However, particularly in GI tract neoplasms, FDG-PET may give false positive results. Therefore, new PET tracers, like 18F-fluoro-thymidine, are being tested and the results look promising. ${ }^{3}$ In response evaluation, all of abovementioned techniques may play a role, but (at least in gastric lymphoma) histopathological assessment is still recommended. ${ }^{7}$

\section{Risk factors}

The most important risk factor in gNHL is Helicobacter pylori (H. pylori) infection. It is considered crucial in MALT pathogenesis, but also probably plays a role in DLBCL and BL growth. Another infectious factor is Campylobacter jejuni (C. jejuni) colonization, which plays a role in immunoproliferative small intestinal disease (IPSID). ${ }^{8}$ Until recently, human immunodeficiency virus (HIV) was considered a significant risk factor for gNHL. Nowadays, effective antiretroviral treatment of HIV patients resulted in lymphoma frequency reduction in these patients. Moreover, OS in HIV lymphoma patients does not differ significantly from corresponding immunocompetent patients. The estimated risk of gNHL in HIV carriers is not substantially different from the average population risk. Other immunodeficiency disorders are connected with a higher gNHL risk. ${ }^{8}$ It should also be noted that inflammatory diseases, even though not caused by infections, increase the risk of gNHL, and celiac disease (CD) is a quintessential example of this process. ${ }^{8}$

\section{Staging and prognosis}

Ann Arbor staging system does not illustrate the exact clinical stage of the disease and is not valuable in prognosis. The most widely used classification is the Lugano system (Table 1).

Marginal zone lymphoma cases comprise over $50 \%$ of primary gNHL cases. ${ }^{1}$ It is seen less commonly in the intestines (5\% of intestine lymphomas) and colorectal area (25\% of colorectal lymphomas). ${ }^{4}$ It usually affects patients over 50 years of age, with a slight male prevalence (1.5:1). Strong evidence on the association between $H$. pylori and gastric MALT (gMALT) has been shown. ${ }^{1}$ Gastric MALT is usually diagnosed in the early stage: typically, an endoscopy reveals multifocal superficial lesions of the mucosa and most patients present with stage I or II disease (Lugano staging system) while intestinal MALT might infiltrate to the intestinal wall. In the case of intestinal MALT, a differential diagnosis has to include the distinction from reactive lymphoid hyperplasia, which may sometimes mimic neoplastic process. ${ }^{4}$ Independently of stage $H$. pylori, eradication therapy should be given to all gMALT patients. Anti-helicobacter regimens contain the following: double antibiotic therapy (clarithromycin + metronidazole or amoxicillin) and proton pump inhibitor. The outcome of the eradication therapy should be evaluated after at least 6 weeks with urea breath test or stool antigen test. It is reasonable to wait for at least 12 months before starting the treatment in patients who achieved endoscopic or clinical response together with $H$. pylori eradication. It is worth remembering that patients with $\mathrm{t}(11 ; 18)(\mathrm{p} 21 ; \mathrm{p} 21)$ are unlikely to respond to $H$. pylori eradication. On the other hand, even $H$. pylori negative gMALT patients might respond to $H$. pylori eradication. In patients who do not achieve a lymphoma regression following antibiotic therapy and have localized disease, irradiation should be applied. In generalized disease immunochemotherapy is highly effective. ${ }^{9}$

Primary GI diffuse large B-cell lymphoma, similarly to MALT, is most typically located in the stomach with a prevalence estimated at $30-40 \%$ of gastric lymphomas. ${ }^{10}$

Table 1. Gastrointestinal lymphoma staging systems

\begin{tabular}{|c|c|c|c|}
\hline Lugano & Paris staging system & Tumor extension & Ann Arbor \\
\hline $\begin{array}{l}\text { St I - confined to the GI tract } \\
\text { (single primary or multiple, non-contiguous) }\end{array}$ & $\mathrm{T} 1-2 \mathrm{NO} \mathrm{MO}$ & mucosa, submucosa, muscularis propria, serosa & $l_{E}$ \\
\hline $\begin{array}{l}\text { St } I \text { - extending into abdomen } \\
\|_{1} \text { - local nodal involvement } \\
\|_{2} \text { - distant nodal involvement }\end{array}$ & $\begin{array}{l}\mathrm{T} 1-3 \mathrm{~N} 1 \mathrm{M0} \\
\mathrm{T} 1-3 \mathrm{~N} 2 \mathrm{M0}\end{array}$ & $\begin{array}{l}\text { regional lymph nodes } \\
\text { more distant regional nodes }\end{array}$ & $\|_{E}$ \\
\hline $\begin{array}{l}\text { St } \|_{E} \text { - penetration of serosa to involve adjacent } \\
\text { organs or tissues }\end{array}$ & T4 NO-2 MO & $\begin{array}{l}\text { invasion of adjacent structures with or without } \\
\text { abdominal lymph nodes }\end{array}$ & $\|_{E}$ \\
\hline $\begin{array}{l}\text { St IV - disseminated extranodal involvement } \\
\text { or concomitant supra-diaphragmatic nodal } \\
\text { involvement }\end{array}$ & $\begin{array}{l}\mathrm{T} 1-4 \mathrm{~N} 3 \mathrm{M0} \\
\mathrm{T} 1-4 \mathrm{~N} 0-3 \mathrm{M} 1 \\
\mathrm{~T} 1-4 \mathrm{~N} 0-2 \mathrm{M} 2 \\
\mathrm{~T} 1-4 \mathrm{~N} 0-3 \mathrm{M} 0-2 \mathrm{Bx} \\
\mathrm{T} 1-4 \mathrm{~N} 0-3 \mathrm{MO}-2 \mathrm{B0} \\
\mathrm{T} 1-4 \mathrm{~N} 0-3 \mathrm{M} 2 \mathrm{~B} 1\end{array}$ & $\begin{array}{c}\text { extra-abdominal lymph nodes } \\
\text { additional distant (non-continuous) gastrointestinal sites } \\
\text { non-gastrointestinal sites } \\
\text { bone marrow not assessed } \\
\text { bone marrow not involved } \\
\text { bone marrow involved }\end{array}$ & \|\|$_{E} \mathrm{i} I \mathrm{~V}$ \\
\hline
\end{tabular}


At the same time, DLBCL is the most common intestinal lymphoma. ${ }^{4}$ Most DLBCLs occur in patients in $6^{\text {th }}$ decade of life, with a male predominance. Some evidence suggests the role of atrophic gastritis, especially among immunocompromised patients, in the etiopathology of gastric DLBCL (gDLBCL). ${ }^{10}$ As with other DLBCL locations, gDLBCL may arise de novo or from transformation of indolent lymphoma, mainly MALT. De novo DLBCLs are bcl 2 and CD10 positive whereas transformed MALT are bcl 2 and CD10 negative. ${ }^{1}$ Generally speaking, c-myc rearrangements are more common in GI aggressive lymphomas than in nodal lymphomas; in DLBCL they account for $10-45 \%$ of cases. But contrary to nodal lymphomas, c-myc rearrangements do not seem to influence negatively the overall survival (OS) ${ }^{11}$ Gastrointestinal DLBCL is usually diagnosed in the early stage, with no bone marrow infiltration and low or intermediate the International Prognostic System (IPI). Presumably, outcomes of treatment are better compared to other extranodal and nodal DLBCL. In a retrospective analysis conducted by López-Guillermo et al., 5-year OS in gDLBCL was $62 \%$ compared to $52 \%$ in the whole DLBCL group. ${ }^{12}$ In another analysis, although OS benefits were not proven, prolonged the Progression Free Survival (PFS) was observed in gDLBCL. ${ }^{13}$ In the era of chemoimmunotherapy, radiotherapy does not improve OS. ${ }^{14,15}$ On the basis of small prospective trials, some authors suggest to start with $H$. pylori eradication only in limited stage $H$. pylori(+) gDLBCL. It concerns both primary and transformed MALT DLBCL if only negative risk factors are not present. ${ }^{14,16}$

Gastrointestinal involvement in MCL is common. The reported frequency is $10-30 \%$. Furthermore, in all probability the data is underestimated. Romaguera et al. conducted an endoscopy in $60 \mathrm{MCL}$ patients. Histopathological involvement of lower GI tract was revealed in 53 patients (88\%) and upper GI tract lesions were found in 28 patients (43\%). ${ }^{17}$ Only 14 (26\%) patients presented with clinical GI symptoms. Significant GI tract histopathological involvement usually does not alter treatment schedule. ${ }^{17}$ European Society for Medical Oncology (ESMO)guidelines recommend an endoscopy in limited stages I/II to exclude asymptomatic involvement. ${ }^{18}$ The most common GI tract involvement manifestation is multiple lymphomatous polyposis. ${ }^{17}$ It is worth remembering that PET-CT might give false negative results and fail to reveal lymphomatous polyposis. ${ }^{19}$ Primary GI MCL is very rare and accounts for only $2 \%$ of primary gNHLs. ${ }^{20}$ Primary GI MCL is usually very aggressive, with high MIPI scores. Survival, compared to nodal MCL involving GI, is poor. As the majority of patients are not autologus stem cel transplantation-eligible, rituximab maintenance is suggested to sustain treatment effects. ${ }^{20}$

Immunoproliferative small intestinal disease (IPSID), formerly known as heavy alpha chain disease, is a rare variant of intestinal MALT lymphoma. It constitutes for 30\% of all GI lymphomas in the Middle East. In the Western countries, it can be diagnosed among immigrants from the Middle East. Median age at the moment of the diagnosis is 20-30 years. Recurrent $C$. jejuni infection role in pathogenesis is suspected. In contrast with other infectious agents, $C$. jejuni colonization is not permanent; moreover, there is no evidence that $C$. jejuni plays a role in cancer development. ${ }^{21}$ In some cases, successful antibiotic therapy may lead to remission, but in other patients transformation to DLBCL was observed. ${ }^{1}$

Primary extranodal FL is rare. The most common location for gastric FL (gFL) is duodenum. ${ }^{22}$ Typically primary intestinal FL is an indolent lymphoma, often limited and with low histological grade (G1-G2). It predominantly affects middle-aged women. Incidental diagnosis in asymptomatic patients undergoing endoscopy for unrelated symptoms is very common. ${ }^{4}$ The most common form of the disease is mucosal polyp. ${ }^{4}$ The tumor has a favorable prognosis even when the disease is disseminated. The indolent course of the disease is considered similar to nodal FL. In the early stages, there is no need to introduce the treatment. ${ }^{22,23}$

Burkitt lymphoma is usually diagnosed in the form of a mass located predominantly in the ileocecal region. Due to its aggressive nature and chemosensitivity, the standard approach is aggressive chemotherapy. Rituximab addition is more widely recommended in recent years. ${ }^{24,25}$ There are casuistic reports on $H$. pylori eradication efficacy in gastric $\mathrm{BL}(\mathrm{gBL})$ therapy. $^{26}$

Lymphomatoid granulomatosis (LG) typically involves the lungs and is rarely found in GI tract. It is an angiodestructive $\mathrm{EBV}(+)$ lymphoma with aggressive course and poor prognosis (OS below 2 years). ${ }^{1}$

Plasmablastic lymphoma (PBL) is an aggressive variant of DLBCL usually diagnosed in immunocompromised patients, particularly $\mathrm{HIV}(+)$. The most common location is the oral cavity. It may also be found in other GI tract parts, primarily in the anal canal. ${ }^{4}$

Primary effusion lymphoma (PEL) is actually a rare form of highly aggressive "plasmablastic" DLBCL arising mainly in immunocompromised patients. Approximately $30 \%$ of extracavitary PEL are diagnosed in the GI tract. ${ }^{4}$

Post-transplant lymphoproliferative disorders (PTLD) are diagnosed in transplant recipients. Gastrointestinal tract involvement may be the primary location or part of the disseminated disease. The most commonly affected part of the GI tract is the distal segment of small intestine.

\section{Mature T-cell lymphomas}

Enteropathy-associated T-cell lymphoma is a rare type of peripheral T-cell lymphoma. Celiac disease is the most common food intolerance in Europe and accounts for 0.5$1 \%$ of EATL cases. Refractory CD appears when the patients fail to improve on a gluten-free diet (2-5\%). Intraepithelial monoclonal lymphocyte proliferation might arise in refractory CD that leads to EATL. The EATL prevalence 
in Western Europe is about 0.14/100,000. It accounts for $1.4 \%$ of NHL cases and $10-25 \%$ of primary intestinal lymphomas. Usually, the diagnosis is made in the patent's $6^{\text {th }}$ decade of life. Men and women are affected with similar frequency. ${ }^{27}$ Clinical symptoms are the consequence of malabsorption with abdominal pain, but many patients present with acute symptoms, such as intestinal bleeding, perforation and obstruction. Although EATL usually appears in refractory $C D$, it may be diagnosed in wellcontrolled CD and even in previously untreated, healthy people. Enteropathy-associated T-cell lymphoma may be localized in every GI tract part but the most common location is the jejunum. Commonly, it manifests as multiple ulcers, tumors and strictures. ${ }^{28}$

The EATL I type concerns 80-90\% of cases. Lymphoma cells derive from $C D$ with villous atrophy and crypt hyperplasia. Tumor cells are medium-sized to large and pleomorphic; reactive inflammatory infiltrate is common and even necrosis might be present. Cells are frequently CD30-positive (which leads to therapeutic implications). The EATL II type is most common in Asia. Very often it does not follow CD. Tumor cells are monomorphic, small to medium-sized. Neither inflammatory infiltrations nor necrosis is observed. Lymphoma cells are DC 30-negative. ${ }^{4,29}$

Conventional chemotherapy based on anthracyclines effects is not satisfactory. Median 5 -year OS is $8-20 \% .^{30}$ The idea of surgical treatment was to debulk the disease and excise tumor masses with high risk of obstruction or perforation during chemotherapy, but so far, surgical treatment did not improve the response. ${ }^{31}$ There is no specific prognostic index for EATL. It seems that low IPI correlates with better OS, ${ }^{30}$ but according to some authors, more PIT is more accurate in EATL risk stratification. ${ }^{29}$ Single risk factors that might be relevant for PFS and OS are: tumor size $>5 \mathrm{~cm}$, poor performance status, high CRP, and high LDH. ${ }^{29}$

Sieniawski et al. introduced intensive IVE/MTX (ifosfamide, epirubicin, etoposide/methotrexate) regimen in 26 ASCT-eligible EATL patients. The patients received 1 cyclophosphamide, doxorubicin, oncovin, prednisone (CHOP) course, 3 ifosfamide, epirubicin, etoposide (IVE) courses and 1 intermediate dose methotrexate course. Chemotherapy was followed by ASCT procedure. The outcome of the patients treated in this protocol was better than median OS achieved after standard chemotherapy. Briefly, $65 \%$ of patients achieved CR vs $42 \%$ in the control group ( $\mathrm{p}=0.06$ ); $39 \%$ of the patients died (including $31 \%$ lymphoma-related deaths), whereas in the control group, $81 \%$ died (61\% died of the EATL) ( $\mathrm{p}=0.001$ and 0.005 , respectively). High response rate correlated with 5 -year OS benefits: $60 \%$ IVE/MTX-treated patients achieved 5 -year OS vs $22 \%$ of patients in the control group $(\mathrm{p}=0.003)$. This is symptomatic that in IVE/MTX only 1 partial remission was obtained and there were no partial remission (PR) in the control group. These results seem to be indicative of the aggressive character of the disease and are arguments for aggressive first-line treatment. IVE/MTX-ASCT regimen may lead to potentially severe toxic complications, such as the following: myelotoxicity, encephalopathy, sepsis, and renal impairment. ${ }^{28}$ There is more data for aggressive approach in EATL. A retrospective study was conducted by European Society for Blood and Marrow Transplantation (EBMT): 44. EATL patients that underwent ASCT consolidation in 2000-2010 were analyzed. First line regimens were heterogeneous: schedules based on anthracyclines, methotrexate, and ifosfamide. More than $50 \%$ of the patients were treated with more than 1 chemotherapy line before ASCT. Thirty-one patients (70\%) were in first complete remission (CR) or PR at the time of the ASCT. Age, gender, disease stage, and B-cell symptoms at diagnosis were not associated with significant PFS or OS differences. The authors concluded that ASCT conducted in first CR/PR is the most effective treatment. Four-year OS in this group was $66 \%$ vs $35 \%$ in the remaining patients $(\mathrm{p}=0.62)$. However, according to the authors, only $50 \%$ of the patients, due to their age, performance status are ASCT-eligible. ${ }^{32}$ There are attempts to introduce new drugs in EATL treatment. Khalaf et al. described brentuximab vedotin efficacy in a EATL patient who was $\mathrm{CD}^{+}{ }^{+}$highly positive. Very good partial remission was observed after 3 cycles. Complete remission was achieved after 8 cycles and sustained during 9-month observation. The most important side effect observed during treatment was exacerbation of neuropathy, which was present at the beginning of the therapy. The authors suggest that brentuximab might be an option for the patients with poor tolerance of more intensive chemotherapy. ${ }^{33}$ Sibon et al. added brentuximab to other regimens. The patients, after achieving remission, underwent ASCT procedure. Preliminary data is very encouraging. ${ }^{34}$ The data on positive effects of alemtuzumab addition to chemotherapy is anecdotal. Furthermore, no durable effects were achieved with this drug in EATL treatment. Available data on RIC-allogenic SCT (sibling) efficacy is also sparse. ${ }^{31}$

Considering the poor prognosis and low chemotherapy effectiveness, there have been attempts to introduce preemptive treatment in $\mathrm{CD}$, i.e., cladribine. However, the safety and efficacy data are very sparse. ${ }^{31}$

Extranodal NK/T-cell lymphoma of the nasal type (ENKTL) is usually located in the nasopharyngeal region. However, sometimes it occurs in various parts of the GI tract. ${ }^{1}$ Virtually always ENKTL is associated with EBV infection. ${ }^{1}$ Differential diagnosis should be made between ENKTL and NK enteropathy (which is a benign GI proliferation) or indolent T-cell lymphoproliferative disease of the GI tract, which are both very rare.

\section{Summary}

Lymphoproliferative disorders of the GI tract are not common and primary GI lymphomas are rare. Gastrointestinal lesions, when found in lymphoma patients, should always be verified and differential diagnosis with other 
diseases should be done (Table 2). Though new imaging techniques are developing rapidly, endoscopy is still the most important diagnostic tool in gastrointestinal lymphomas. Histopathological type may vary, with the 2 most common morphologic subtypes being MALT and DLBCL. The most typical location of GI lymphomas is the stomach. The discovery of association of $H$. pylori infection to gastric lymphoma led to serious approach modificatin in this disease. Nowadays, in the antiretroviral HAART era, HIV seems to lose its importance as a risk factor. T-cell lymphomas are more aggressive than B-lineage NHL and there is still much to do to improve patient outcome.

Table 2. Differential diagnosis of gastrointestinal lymphomas

\begin{tabular}{|l|}
\hline Gl lymphomas - differential diagnosis \\
Crohn disease \\
Adenocarcinoma and other solid tumors \\
Benign lymphoid hyperplasia \\
Peptic ulcer disease \\
Celiac disease \\
Bacterial and fungal infections of Gl tract \\
\hline
\end{tabular}

\section{References}

1. Bautista-Quach MA, Ake CD, Chen M, Wang J. Gastrointestinal lymphomas: Morphology, immunophenotype and molecular features. $J$ Gastrointest Oncol. 2012;3(3):209-225.

2. Sieniawski M, Angamuthu N, Boyd K, et al. Evaluation of enteropathy-associated T-cell lymphoma comparing standard therapies with a novel regimen including autologous stem cell transplantation. Blood. 2010;115(18):3664-3670.

3. Ghimire P, Wu GY, Zhu L. Primary gastrointestinal lymphoma. World J Gastroenterol. 2011;17(6):697-707.

4. Foukas PG, de Leval L. Recent advances in intestinal lymphomas. Histopathology. 2015;66(1):112-136.

5. Janssen J. The impact of EUS in primary gastric lymphoma. Best Pract Res Clin Gastroenterol. 2009;23(5):671-678.

6. Byun JH, Ha HK, Kim AY, et al. CT findings in peripheral T-cell lymphoma involving the gastrointestinal tract. Radiology. 2003;227(1):59-67.

7. Boot $\mathrm{H}$. Diagnosis and staging in gastrointestinal lymphoma. Best Pract Res Clin Gastroenterol. 2010;24(1):3-12.

8. Andrews CN, John Gill M, Urbanski SJ, Stewart D, Perini R, Beck P. Changing epidemiology and risk factors for gastrointestinal nonHodgkin's lymphoma in a North American population: Populationbased study. Am J Gastroenterol. 2008;103(7):1762-1769.

9. Zucca E, Copie-Bergman C, Ricardi U, Thieblemont C. Raderer M, Ladetto M; on behalf of the ESMO Guidelines Working Group. Gastric marginal zone lymphoma of MALT type: ESMO Clinical Practice Guidelines for diagnosis, treatment and follow-up. Ann Oncol. 2013; 24(Suppl 6):144-148.

10. Martinelli G, Gigli F, Calabrese L, et al. Early stage gastric diffuse large B-cell lymphomas: Results of a randomized trial comparing chemotherapy alone versus chemotherapy + involved field radiotherapy. Leuk Lymphoma. 2009;50(6):925-931.

11. Choi SY, Kim SJ, Kim WS, Kim K, Ko YH. Aggressive B cell lymphomas of the gastrointestinal tract: Clinicopathologic and genetic analysis. Virchows Arch. 2011;459(5):495-502.

12. López-Guillermo A, Colomo L, Jiménez M, et al. Diffuse large B-cell lymphoma: Clinical and biological characterization and outcome according to the nodal or extranodal primary origin. $J$ Clin Oncol. 2005;23(12):2797-2804.
13. Nakajima Y, Tomita N, Itabashi M, et al. Analysis of outcomes in patients with supra-diaphragmatic vs infra-diaphragmatic diffuse large B cell lymphoma treated with R-CHOP therapy. Leuk Res. 2015;39(2):198-203.

14. Cuccurullo R, Govi S, Ferreri AJM. De-escalating therapy in gastric aggressive lymphoma. World J Gastroenterol. 2014;20(27):8993-8997.

15. Avilés A, Nambo M.J, Neri N, et al. The role of surgery in primary gastric lymphoma: Results of a controlled clinical trial. Ann Surg. 2004; 240(1):44-50.

16. Kuo SH, Yeh KH, Wu MS, et al. Helicobacter pylori eradication therapy is effective in the treatment of early-stage $H$. pylori-positive gastric diffuse large B-cell lymphomas. Blood. 2012;119(21):4838-4844.

17. Romaguera JE, Medeiros LJ, Hagemeister FB, et al. Frequency of gastrointestinal involvement and its clinical significance in mantle cell lymphoma. Cancer. 2003;97(3):586-591.

18. Dreyling M, Geisler C, Hermine O, et al; ESMO Guidelines Working Group. Newly diagnosed and relapsed mantle cell lymphoma: ESMO Clinical Practice Guidelines for diagnosis, treatment and follow-up. Ann Oncol. 2014;25(Suppl 3):iii83-92. doi:10.1093/annonc/mdu264

19. Saito M, Miyazaki M, Tanino $M .{ }^{18}$ F-FDG PET/CT imaging for a gastrointestinal mantle cell lymphoma with multiple lymphomatous polyposis. World J Gastroenterol. 2014;20(17):5141-5146.

20. Dasappa L, Suresh Babu MC, et al. Primary gastrointestinal mantle cell Iymphoma: A retrospective study. J Gastrointest Cancer. 2014;45(4): 481-486.

21. Zucca E, Bertoni F, Vannata B, Cavalli F. Emerging role of infectious etiologies in the pathogenesis of marginal zone B-cell lymphomas. Clin Cancer Res. 2014;20(20):5207-5216.

22. Misdraji J, Harris NL, Hasserjian RP, Lauwers GY, Ferry JA. Primary follicular lymphoma of the gastrointestinal tract. Am J Surg Pathol. 2011; 35(9):1255-1263.

23. Damaj G, Verkarre V, Delmer A, et al. Primary follicular lymphoma of the gastrointestinal tract: A study of 25 cases and a literature review. Ann Oncol. 2003;14(4):623-629.

24. Perkins AS, Friedberg JW. Burkitt lymphoma in adults. Hematology Am Soc Hematol Educ Program. 2008:341-348.

25. Hoelzer D, Walewski J, Döhner H, et al; German Multicenter Study Group for Adult Acute Lymphoblastic Leukemia. Improved outcome of adult Burkitt lymphoma/leukemia with rituximab and chemotherapy: Report of a large prospective multicenter trial. Blood. 2014; 124(26):3870-3879.

26. Baumgaertner I, Copie-Bergman C, Levy M, et al. Complete remission of gastric Burkitt's lymphoma after eradication of Helicobacter pylori. World J Gastroenterol. 2009;15(45):5746-5750.

27. Zettl A, deLeeuw R, Haralambieva E, Mueller-Hermelink HK. Enteropathy-type T-cell lymphoma. Am J Clin Pathol. 2007;127(5):701-706.

28. Sieniawski M, Angamuthu N, Boyd K, et al. Evaluation of enteropathy-associated T-cell lymphoma comparing standard therapies with a novel regimen including autologous stem cell transplantation. Blood. 2010;115(18):3664-3670.

29. Delabie J, Holte H, Vose JM, et al. Enteropathy-associated T-cell lymphoma: Clinical and histological findings from the international peripheral T-cell lymphoma project. Blood. 2011;118(1):148-155.

30. Nijeboer P, de Baaij LR, Visser O, et al. Treatment response in enteropathy associated T-cell lymphoma: Survival in a large multicenter cohort. Am J Hematol. 2015;90(6):493-498.

31. Di Sabatino A, Biagi F, Gobbi PG, Corazza GR. How I treat enteropathy-associated T-cell lymphoma. Blood. 2012;119(11):2458-2468.

32. Jantunen $\mathrm{E}$, Boumendil A, Finel $\mathrm{H}$, et al; Lymphoma Working Party of the EBMT. Autologous stem cell transplantation for enteropathy-associated T-cell lymphoma: A retrospective study by the EBMT. Blood. 2013;121(13):2529-2532.

33. Khalaf WF, Caldwell ME, Reddy N. Brentuximab in the treatment of CD30-positive enteropathy-associated T-cell lymphoma. J Natl Compr Canc Netw. 2013;11(2):137-140.

34. Sibon D, Malamut G. Enteropathy-associated T-cell lymphoma type I, but not refractory celiac disease, strongly expresses CD30 and might benefit from brentuximab. Vedotin Blood. 2013;122:4252. 